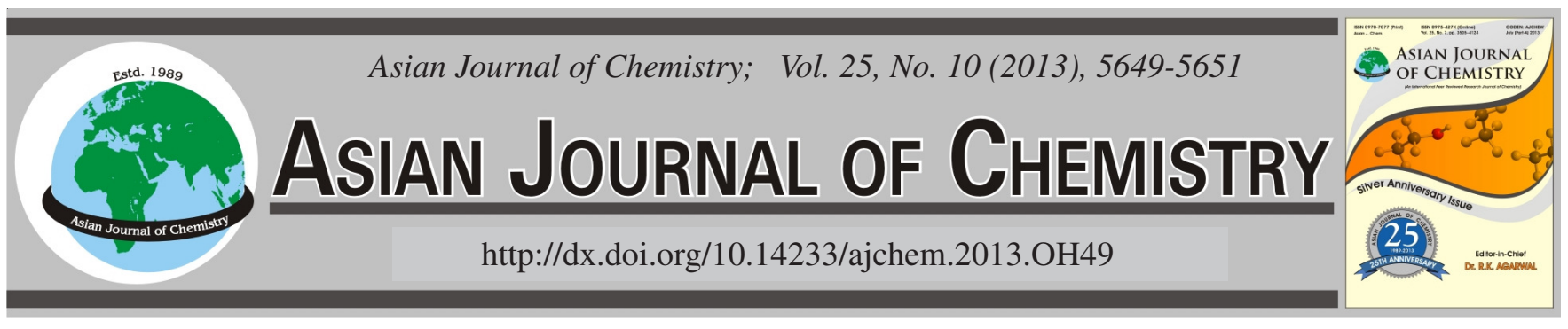

\title{
Preparation and Property of Hydroxyapatite/ \\ Poly(methyl methacrylate) Composites by in situ Copolymerization $\dagger$
}

\author{
Benhong YAnG ${ }^{*}$, Meng Li, Yun Wu and Fei Wang \\ Department of Chemistry and Materials Engineering, Hefei University, Hefei 230601, P.R. China \\ *Corresponding author: E-mail: yangbh@hfuu.edu.cn
}

AJC-13292

\begin{abstract}
Modified hydroxyapatite (m-HA) nanoparticles were synthesized via the hydrothermal method and followed by modification with a ilane coupling agent. Hydroxyapatite/poly(methyl methacrylate) composites were prepared using the in situ copolymerization of methyl methacrylate and modified hydroxyapatite particles. SEM images revealed that modified hydroxyapatite particles dispersed homogeneously in poly(methyl methacrylate) matrix when its content was lower than $1.0 \mathrm{wt} \%$ due to the covalent interaction between modified hydroxyapatite and poly(methyl methacrylate), but tended to aggregate at higher contents. Results show that the incorporation of modified hydroxyapatite could improve thermal and mechanical properties of poly(methyl methacrylate).
\end{abstract}

Key Words: Hydroxyapatite, Poly(methyl methacrylate), Composites, Properties.

\section{INTRODUCTION}

In recent years, organic-inorganic composites for some specific functional applications, in particular, for bone tissue engineering $^{1-3}$, have received much attention. Natural bone is an innate example of inorganic-organic biocomposite, which primarily consists of collagen and hydroxyapatite $\left[\left(\mathrm{Ca}_{10}\left(\mathrm{PO}_{4}\right)_{6}\right.\right.$ $(\mathrm{OH}), \mathrm{HA}]$. As the mineral component of bone, hydroxyapatite is a suitable biomaterial for bone repair due to its biocompatibility and osteoconductivity. However, application of hydroxyapatite in this area has been limited by the poor processability and inherent brittleness. To combine osteoconductivity of hydroxyapatite and biocompatibility of polymers, hydroxyapatite/polymer composites have been developed for bone tissue engineering to mitigate the inherent brittleness and poor fracture toughness of hydroxyapatite while providing additional function either by physical mixing ${ }^{3}$ or by in situ processing ${ }^{4,5}$.

Basically, the big issue in this area is how to disperse hydroxyapatite homogeneously into polymer matrix. It is conceivable that surface modification of hydroxyapatite particles and the in situ polymerization benefit incorporation of hydroxyapatite particles ${ }^{6,7}$. In this study, hydroxyapatite/poly(methyl methacrylate) composites were prepared by the in-suit bulk polymerization of methyl methacrylate, which was chosen as a matrix owing to its favourable biocompatibility and low toxicity. The structure-property relationship for hydroxyapatite/poly(methyl methacrylate) composites is especially discussed here.

\section{EXPERIMENTAL}

A solution of ammonium dihydrogen phosphate was added dropwise into a calcium chloride solution at the stoichiometric ratio $(\mathrm{Ca}: \mathrm{P}=1.67: 1)$ with constant stirring. The $\mathrm{pH}$ value was adjusted to 12 by addition of ammonia. Then, CTAB (1.0 wt \%) was added into the mixture with constant stirring for $0.5 \mathrm{~h}$. The resultant suspension in the flask was poured into Teflon-lined stainless steel autoclaves, sealed and maintained at $160{ }^{\circ} \mathrm{C}$ for $10 \mathrm{~h}$ in an electric oven. The final solid product was filtered out and washed with distilled water twice, dried at $60^{\circ} \mathrm{C}$ in air for $24 \mathrm{~h}$ and finally grinded to powder. The as-prepared hydroxyapatite particles were treated with silane coupling agent (vinyltriethoxysilane) via a sol-gel process to graft vinyl groups on the surface of hydroxyapatite.

Hydroxyapatite/poly(methyl methacrylate) composites with different content of modified hydroxyapatite particles were prepared by in situ bulk polymerization of methyl methacrylate monomer, in which modified hydroxyapatite particles dispersed uniformly. The polymerization was initiated by AIBN. After prepolymerization at $80^{\circ} \mathrm{C}$ for $1 \mathrm{~h}$, the solution was transferred into a mould and kept at $80^{\circ} \mathrm{C}$ for $4 \mathrm{~h}$ to give sample bars.

\section{RESULTS AND DISCUSSION}

The XRD spectra of the pristine hydroxyapatite and modified hydroxyapatite particles are shown in Fig. 1. Fully consistent with the standard data (JCPDS No. 09-0432) of

$†$ Presented to the 6th China-Korea International Conference on Multi-functional Materials and Application, 22-24 November 2012, Daejeon, Korea 
hydroxyapatite, typical diffraction peaks of hydroxyapatite particles such as (002), (210), (211), (300), (310), (222) and (213) could be found in both spectra, meaning that surfacemodification did not change the structure of hydroxyapatite. Further, the crystallinity of hydroxyapatite has little change after saline grafting onto the surface of pristine hydroxyapatite particles.

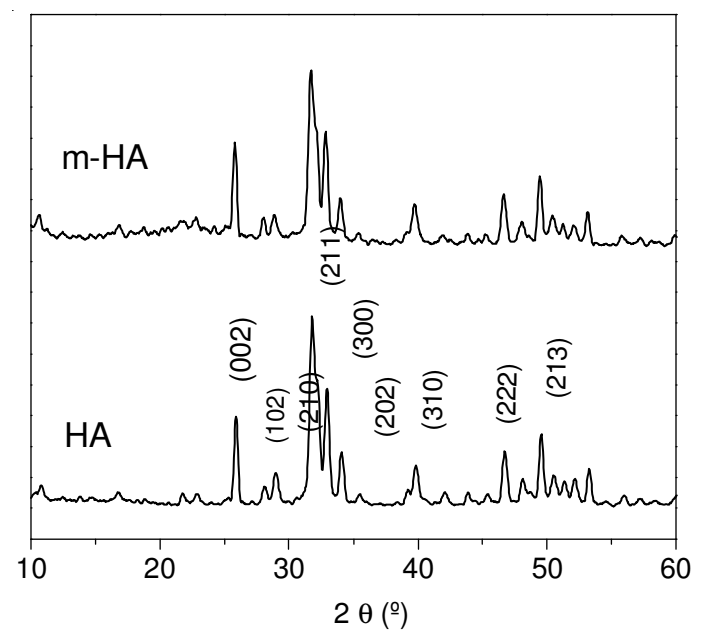

Fig. 1. XRD patterns of pure hydroxyapatite and modified hydroxyapatite particles

SEM images of pristine hydroxyapatite, modified hydroxyapatite and hydroxyapatite/poly(methyl methacrylate) composites are displayed in Fig. 2. Pristine hydroxyapatite particles have a needle-like shape in Fig. 2(a), while modified hydroxyapatite particles exhibited a rougher surface morphology in Fig. 2(b) resulting from the anchoring of silane coupling agents onto the surface of hydroxyapatite particles. Fig. 2(c) indicates that modified hydroxyapatite particles homogeneously disperse in poly(methyl methacrylate) at a low content (1.0 wt \%), whereas Fig. 2(d) shows that modified hydroxyapatite particles tend to agglomerate in larger size at a higher content $(10 \mathrm{wt} \%)$. Even though modified hydroxyapatite particles have a tendency to aggregate, their aggregates are in the similar size and disperse homogeneously in poly(methyl methacrylate) matrix, probably due to covalent interactions.
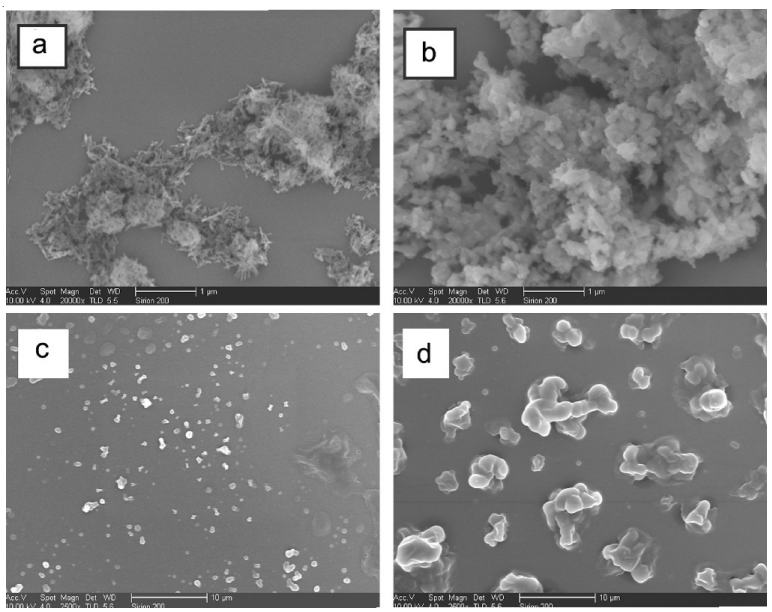

Fig. 2. SEM images of hydroxyapatite (a), modified hydroxyapatite (b), hydroxyapatite/poly(methyl methacrylate) $1.0 \%$ (c) and HA/ PMMA $10 \%$ (d)
Fig. 3 displays the thermogravimetric curves of poly(methyl methacrylate) and its composites with hydroxyapatite particles in a nitrogen environment. Hydroxyapatite/poly(methyl methacrylate) composites have similar thermogravimetric curves to that of poly(methyl methacrylate), all indicating three-step decompositions and stable up to 200 ${ }^{\circ} \mathrm{C}$ and completely degraded at $c a 450{ }^{\circ} \mathrm{C}$, revealing that the incorporation of hydroxyapatite didn't substantively affect the thermal behaviour of poly(methyl methacrylate). However, incorporation of nanosize hydroxyapatite improves the thermal stability. As seen in Fig. 3, the decomposition temperature $\left(\mathrm{T}_{\mathrm{d}}\right.$, defined as the temperature of $5 \%$ weight loss) of HA/PMMA increases with the increase of hydroxyapatite content. For example, HA/PMMA1.0\% has a $\mathrm{T}_{\mathrm{d}}$ of $224.3{ }^{\circ} \mathrm{C}$, which is $15.9{ }^{\circ} \mathrm{C}$ higher than that of poly(methyl methacrylate). This is possibly associated with the strong interaction between hydroxyapatite nanoparticles and poly(methyl methacrylate) chains, which blocked the free motion of polymer chains and delayed the polymer from thermal decomposition.

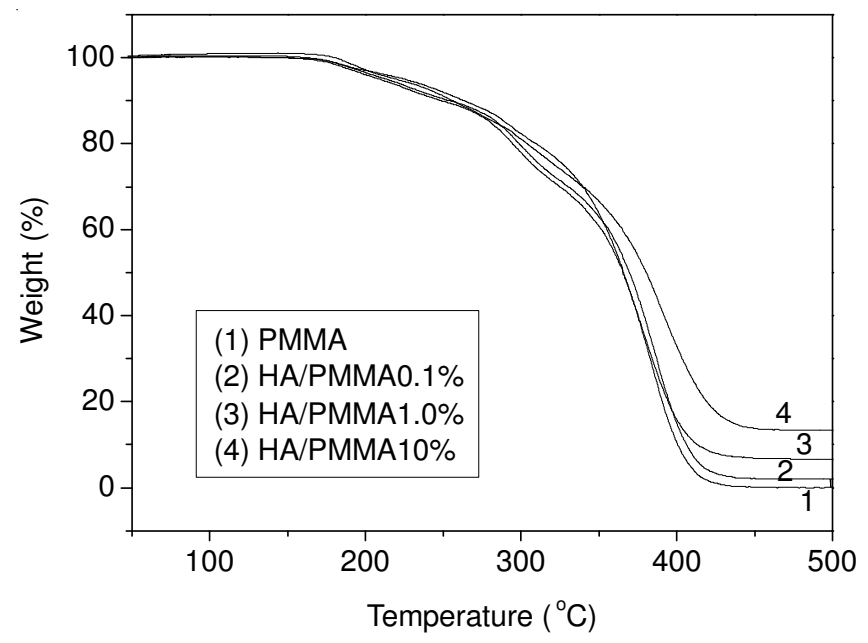

Fig. 3. TGA curves of poly(methyl methacrylate) and HA/PMMA composites with different content of modified hydroxyapatite

Fig. 4 shows the dependence of tensile strength on modified hydroxyapatite content. The tensile strength of composites increased with the content of modified hydroxyapatite ranging from $0.1 \mathrm{wt} \%$ to $1.0 \mathrm{wt} \%$ to a maximum peak at $26 \mathrm{MPa}$. The result indicates that incorporation of modified hydroxyapatite particles played an important role in improving mechanical property of the composite, because of the covalent interaction and its induced uniform dispersion of modified hydroxyapatite aggregations in poly(methyl methacrylate).

\section{Conclusion}

HA/PMMA nanocomposites were prepared by the in situ copolymerization between methyl methacrylate monomers and surface-modified hydroxyapatite nanoparticles. SEM images reveal that hydroxyapatite particles disperse homogeneously in poly(methyl methacrylate) matrix when its content was lower than $1.0 \mathrm{wt} \%$ and tend to aggregate with higher hydroxyapatite content. The incorporation of modified hydroxyapatite could improve thermal and mechanical property of poly(methyl methacrylate) significantly with proper hydroxyapatite contents. 


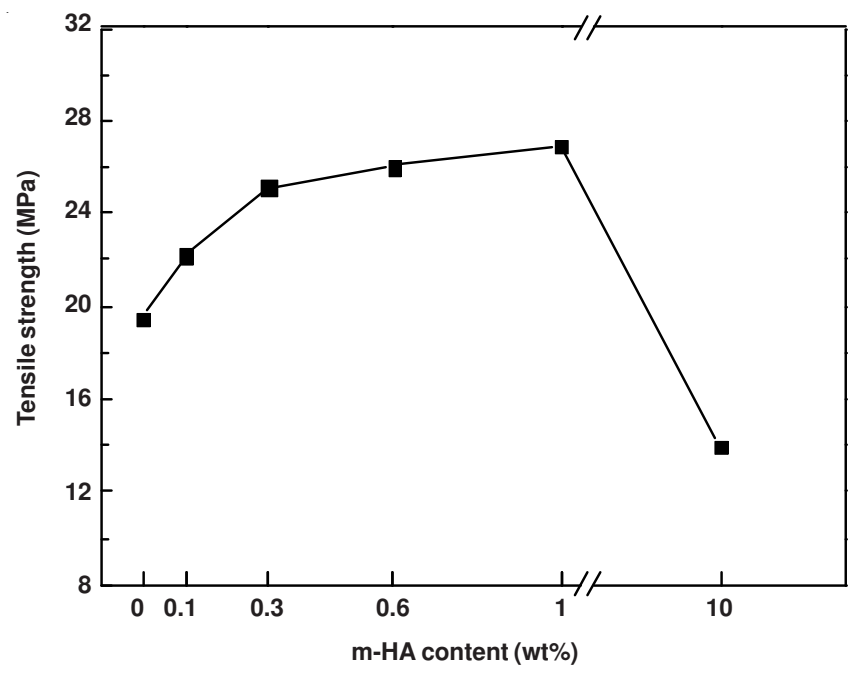

Fig. 4. Effect of modified hydroxyapatite content on the tensile strength of HA/PMMA composites

\section{ACKNOWLEDGEMENTS}

The authors are grateful for the financial support from the Natural Science Foundation of Anhui Educational Department (No.KJ2011A247), the Natural Science Foundation of Anhui Province (No.1208085MB24) and the Natural Science Foundation of Hefei University (No.11KY01ZR).

\section{REFERENCES}

1. K. Rezwan, Q.Z. Chen and J.J. Blaker, Biomaterials, 27, 3413 (2006). 2. S.S. Kima, M.S. Park and O. Jeon, Biomaterials, 27, 1399 (2006).

3. R.K. Roeder, G.L. Converse, R.J. Kane and and W.M. Yue, JOM, 60, 38 (2008).

4. A. Sinha and A. Guha, Mater. Sci. Eng. C, 29, 1330 (2009).

5. W.Y. Choi, H.E. Kim, S.Y. Oh and Y.H. Koh, Mater. Sci. Eng. C, 30, 777 (2010).

6. J.C. Wei, P. He, A.X. Liu, X.S. Chen, X.H. Wang and X.B. Jing, Macromol. Biosci., 9, 1237 (2009).

7. L. Chen, J.M. Mccrate, J.C.M. Lee and H. Li, Nanotechnology, 22, 1 (2011). 